\title{
Características asociadas al absentismo laboral prolongado de causa médica en trabajadores agroindustriales en Perú: un estudio transversal
}

Wilfor Aguirre Quispe ${ }^{1}$, Joan Manuel Moreno Luján ${ }^{2}$

\author{
RESUMEN
}

Objetivo: Describir y analizar los factores asociados al absentismo laboral prolongado de causa médica en trabajadores agroindustriales.

Materiales y métodos: Se realizó un estudio de fuente secundaria en una población de 3150 trabajadores agroindustriales en Perú. Se tomó una muestra de 9443 casos de absentismo laboral durante los años 2012 y 2013. Se definió absentismo laboral prolongado como $\geq 10$ días. Los factores analizados fueron edad, sexo, tiempo de trabajo, tipo de actividad, horario de trabajo, estabilidad laboral, tipo de contingencia y sistema afectado según diagnóstico. Se aplicó análisis estadístico descriptivo y multivariado mediante regresión logística binaria.

Resultados: Los factores que explican el absentismo laboral prolongado fueron los accidentes de trabajo (OR: 13,9; IC: $11,2-17,2$ ), horario atípico (OR: 3,14; IC: 1,58-6,25), menor tiempo de servicio (OR: 0,95; IC: 0,95-0,96), actividad laboral moderada-intensa (OR: 8,96; IC: 4,53-17,2) y menor estabilidad laboral (OR: 0,64; IC: 0,54-0,77).

Conclusiones: El tiempo de servicio (>15 años), el horario de trabajo atípico, la actividad física moderada-intensa, los accidentes de trabajo y un contrato de trabajo no estable predicen con alta certeza el absentismo laboral prolongado. El sexo, la edad y el sistema afectado no resultaron ser predictores de absentismo laboral prolongado, pero presentan diferencias relevantes en el análisis bivariado.

Palabras clave: Absentismo; Trabajadores; Accidentes de trabajo; Agroindustria (Fuente: DeCS BIREME).

\section{Characteristics associated with prolonged work absenteeism due to medical reasons among agribusiness workers in Peru: a cross-sectional study}

\section{ABSTRACT}

Objective: To describe and analyze the factors associated with prolonged work absenteeism due to medical reasons among agribusiness workers.

Materials and methods: A secondary source research was conducted in a population of 3,150 agribusiness workers in Peru. A sample of 9,443 cases of work absenteeism was taken during the years 2012 and 2013. Long-term work absenteeism was defined as $\geq 10$ days. Analyzed factors were: age, sex, job tenure, type of activity, work schedule, job stability, type of contingency, and affected body system according to the diagnosis. A descriptive and multivariate statistical analysis was applied by binary logistic regression.

Results: The factors explaining prolonged work absenteeism were occupational accidents (OR: 13.9, Cl: 11.217.2), atypical schedule (OR: 3.14, $\mathrm{Cl}: 1.58-6.25)$, shorter job tenure (OR: 0.95, Cl: 0.95-0.96), moderate-intense work activity (OR: $8.96, \mathrm{Cl}: 4.53-17.2$ ), and lower job stability (OR: 0.64 ; $\mathrm{Cl}: 0.54-0.77$ ).

Conclusions: Job tenure (> 15 years), atypical work schedule, moderate-intense physical activity, occupational accidents, and an unstable job contract predict prolonged work absenteeism with high certainty. Sex, age and the affected body system were not predictive of prolonged work absenteeism, but showed significant differences in the bivariate analysis.

Keywords: Absenteeism; Workers; Occupational accidents; Agribusiness (Source: MeSH NLM).

1. Instituto Nacional de Salud. Lima, Perú.

2. Instituto Nacional de Enfermedades Neoplásicas. Lima, Perú. 


\section{INTRODUCCIÓN}

Según la Organización Internacional del Trabajo (OIT) anualmente ocurren más de 317 millones de accidentes en el trabajo, muchos de los cuales resultan en absentismo laboral. El costo es enorme y la carga económica de las malas prácticas de seguridad y salud en el trabajo representa $4 \%$ del producto bruto interno (PBI) global por año ${ }^{(1)}$.

El absentismo laboral de causa médica es un problema de salud pública mundial por lo que representa una considerable carga económica para la sociedad con importantes consecuencias para las instituciones en términos de pérdida de la productividad, costos de aseguramiento y reemplazo del trabajador ${ }^{(2)}$.

En el Perú se han realizado algunas estimaciones del costo que genera el absentismo laboral en áreas muy específicas (3-5).

Un estudio realizado en trabajadores del área de la salud mostró que el absentismo representa el $21 \%$ del costo generado por enfermedad ${ }^{(5)}$.

Son escasos los estudios descriptivos que han analizado el absentismo laboral, los pocos estudios publicados se centran en trabajadores de la salud, minería y otros $(4,6-8)$. Estos resultados no son generalizables al sector agroindustrial, por lo que existe un vacío de información epidemiológica en esta población.

En Latinoamérica, un estudio sobre absentismo en trabajadores agroindustriales realizado en Brasil (9), evidenció una asociación principalmente a enfermedades del sistema osteomuscular y del tejido conectivo. Datos de áreas distintas a la agroindustria han mostrado relación de ciertos factores con el incremento del absentismo, y su asociación con trastornos musculoesqueléticos, principalmente con lumbalgia ${ }^{(10)}$; el género masculino y la edad también parecen tener una implicancia en el incremento de días de absentismo ${ }^{(11,12)}$. Asimismo, se ha identificado que las cargas pesadas de trabajo físico, posturas de trabajo no ergonómicas o inadecuadas, y el antecedente de absentismo previo, son factores que tienen una influencia limitada, pero consistente, en el riesgo del absentismo laboral (13-15). Respecto a la estabilidad laboral (16) los hallazgos no muestran una clara relación con el absentismo. Otro factor que ha sido estudiado y parece guardar relación con el absentismo es el horario atípico de trabajo ${ }^{(17,18)}$.

Estos factores analizados para otros grupos laborales podrían tener un comportamiento diferente en el sector agroindustrial, por lo que resulta necesario un análisis específico en esta población. Los datos de los servicios médicos de salud ocupacional deberían derivar en la transferencia e incorporación de dicha información para mejorar la vigilancia de la salud de los trabajadores y por tanto mejorar las políticas públicas de salud a nivel nacional.

El presente estudio tiene como objetivo describir y analizar las diferencias entre estos factores en relación a la prolongación de los días de absentismo laboral, lo cual ayudaría a aclarar el panorama de la salud ocupacional en nuestro país.

\section{MATERIALES Y MÉTODOS}

Diseño del estudio

Se realizó un estudio de fuente secundaria, transversal y analítico en trabajadores de una empresa agroindustrial dedicada al cultivo, procesamiento, industrialización, venta de caña de azúcar y derivados en la región La Libertad, Perú.

\section{Población y muestra}

La población analizada fue de 3150 trabajadores. Los datos corresponden a la fuerza laboral de dicha empresa durante los años 2012 y 2013, y fueron recopilados de la base de datos del Servicio de Salud Ocupacional de dicha empresa, la cual utiliza un sistema de información estandarizado que permite el registro de absentismos detallados para seguimiento y control de cada trabajador. El estudio usó los datos de toda la población de trabajadores que presentaron absentismo laboral durante el periodo 2012 2013. Los criterios de inclusión fueron ser trabajador activo de la empresa, hombre o mujer, con edad mayor de 18 años y tiempo laboral mínimo de 6 meses, haber presentado absentismo laboral de causa médica al menos una vez durante el periodo del 01 de enero del 2012 al 31 de diciembre del 2013, contar con diagnóstico definido que generó el absentismo mediante certificado de descanso médico externo o diagnóstico realizado por el médico del Servicio de Salud Ocupacional. Los criterios de exclusión fueron ser parte del personal no activo en la empresa (familiares de trabajadores u otros), absentismo laboral por gestación o lactancia, y falta de datos necesarios en el registro de información.

\section{Variables}

El absentismo laboral se midió en días de ausencia laboral de causa médica. El absentismo prolongado para este estudio se definió como todo absentismo laboral de causa médica mayor o igual a 10 días laborables (14 días calendario) y el absentismo corto, menor a 10 días laborables, corroborado con certificado médico externo o por diagnóstico del Servicio de Salud Ocupacional. 
Las variables independientes fueron edad, sexo, contingencia del absentismo laboral (que puede ser enfermedad común, accidente común y accidente, según la definición del reglamento de la ley 29783 "Ley de Seguridad y Salud en el Trabajo" que rige para nuestro país), sistema del cuerpo afectado según diagnóstico, tiempo de servicio (años), tipo de horario de trabajo (atípico / no atípico), tipo de contrato laboral (estable / no estable) y tipo de actividad física. Este último parámetro se define como actividad física escasa (trabajador que tiene un puesto laboral administrativo), actividad física leve-moderada (cuando la exposición a levantamiento de cargas es esporádica o actividad motora es rutinaria pero realizada en no más del $30 \%$ del tiempo de sus actividades o funciones laborales), y finalmente, actividad física moderada-intensa (trabajo con exposición a levantar cargas con frecuencia o actividad motora es rutinaria y realizada en más del $30 \%$ del tiempo de sus actividades o funciones laborales).

El horario atípico de trabajo se definió como todo aquel que presente horarios rotativos de trabajo alternando entre horarios diurnos y nocturnos, horarios nocturnos exclusivamente o por periodos largos que pudiesen alterar el ritmo circadiano.

\section{Procedimiento}

Los datos del sistema de registro médico para el periodo 2012-2013 fueron obtenidos según los criterios establecidos, se excluyeron los datos que no cumplieron dichos criterios de selección y, finalmente, se obtuvo 9443 registros de absentismos en dicho periodo (Figura 1). Se analizaron los factores descritos en relación al tipo de absentismo. Debido a que se evaluó un periodo de 2 años, hubo trabajadores que presentaron varios absentismos en dicho periodo, por lo que se consideró la suma de los días en quienes el diagnóstico fue repetitivo por enfermedad crónica no resuelta y recidivante, y por separado en quienes la causa del absentismo laboral fue completamente diferente o cuyo diagnóstico repetitivo obedecía a episodios no relacionados.

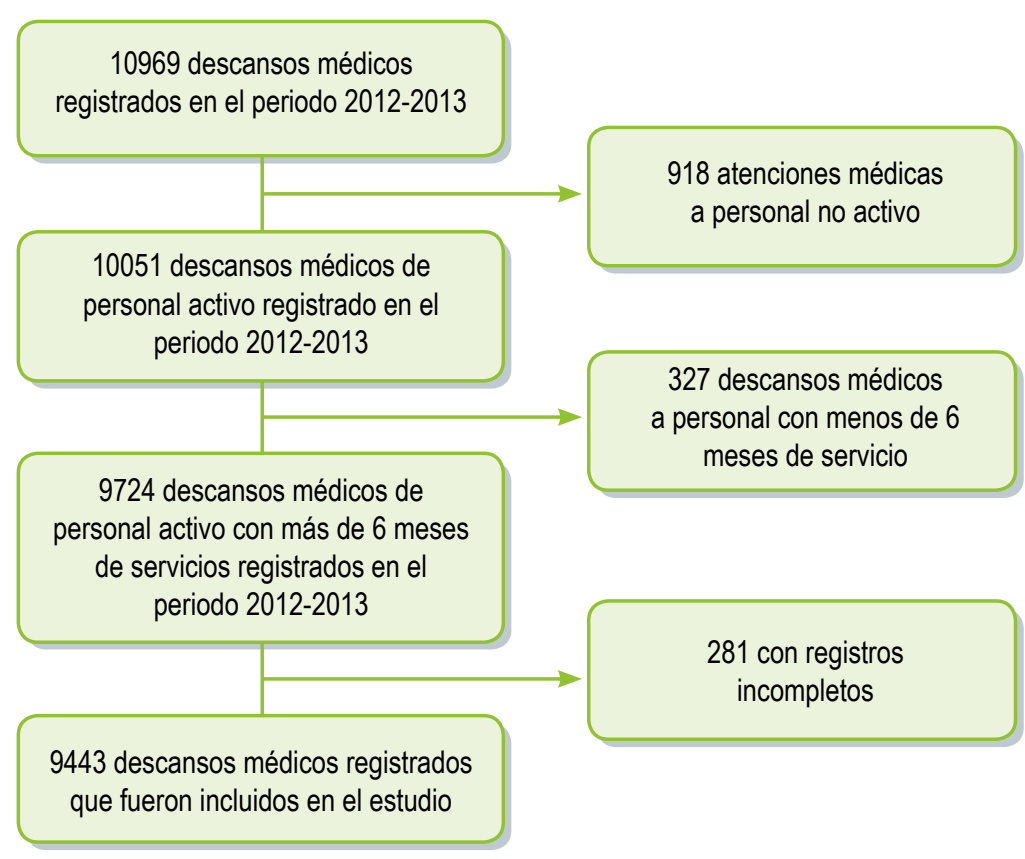

Figura 1. Flujograma de datos seleccionados para el estudio

\section{Análisis estadístico}

Se realizó el análisis descriptivo tanto para variables categóricas como para variables numéricas continuas. Se analizó el absentismo laboral prolongado en relación a las variables independientes categóricas mediante la prueba Chi-Cuadrado, y numéricas continuas mediante t-Student, ambas para un nivel de significancia de 0,05. Posteriormente, se construyó un modelo multivariado utilizando regresión logística binaria. La asociación multivariada entre las 
variables independientes y la variable resultado se realizó mediante la estimación del Odds ratio (OR) multivariado. El modelo fue definido mediante el ingreso progresivo de las variables que habían mostrado significancia estadística en el análisis bivariado y se evaluó su significancia en el modelo mediante el Chi-Cuadrado de Wald. Finalmente, el mejor modelo fue evaluado mediante pruebas de bondad de ajuste y R2 de Cox Snell para dicho modelo. Se utilizó Excel y Stata v.12.0.

\section{Aspectos éticos}

La investigación no involucró participación directa de seres humanos, se trabajó con una fuente de datos secundaria. El manejo de la información recabada fue confidencial, se utilizó códigos para cada trabajador. No se ha consignado el nombre de la empresa agroindustrial de donde se obtuvieron los datos por razones de confidencialidad.

\section{RESULTADOS}

De los 9443 registros de absentismo laboral, se observó que el promedio de días de absentismo laboral fue de 4,24 días con una desviación estándar (DS) de 9,8 días. Al categorizar en tipo de absentismo laboral solo el 6,64 \% presento absentismo prolongado con una media de 27,3 días (DS: 28,9 días).

De la muestra total, el $96,9 \%$ (9154) fue de sexo masculino y, únicamente, el 3,1\% (289), de sexo femenino, presentando absentismo laboral prolongado en un 6,65 \% y 6,23\%, respectivamente (Tabla 1 ).

Tabla 1. Estadística descriptiva de la población del estudio y resultados del análisis bivariado para absentismo laboral prolongado

\begin{tabular}{|c|c|c|c|c|c|c|c|}
\hline Factores & Categorías & $\mathbf{n}$ & $\%$ & $\begin{array}{c}\text { Absentismo } \\
\text { prolongado (\%) }\end{array}$ & $\mathbf{p}$ & OR & I.C. $(95 \%)$ \\
\hline \multirow[t]{2}{*}{ Edad } & $<45$ años & 4791 & 50,74 & 7,89 & $<0,001$ & 1,51 & $1,28-1,79$ \\
\hline & $\geq 45$ años & 4652 & 49,26 & 5,35 & --- & --- & --- \\
\hline \multirow[t]{2}{*}{ Sexo } & Masculino & 9154 & 96,94 & 6,65 & 0,77 & 1,07 & $0,66-1,85$ \\
\hline & Femenino & 289 & 3,06 & 6,23 & --- & --- & --- \\
\hline \multirow{3}{*}{ Tiempo de servicio } & 0-15 años & 5372 & 56,89 & 8,41 & $<0,001$ & 2,04 & $1,70-2,46$ \\
\hline & 15-30 años & 2435 & 25,79 & 2,92 & $<0,001$ & 0,35 & $0,27-0,45$ \\
\hline & > 30 años & 1636 & 17,32 & 6,36 & 0,662 & 0,95 & $0,75-1,18$ \\
\hline \multirow{3}{*}{ Contingencia } & Enfermedad común & 8124 & 86,3 & 5,2 & $<0,001$ & 0,27 & $0,22-0,32$ \\
\hline & Accidente común & 644 & 6,82 & 0,0 & --- & --- & --- \\
\hline & Accidente de trabajo & 675 & 7,15 & 32,4 & $<0,001$ & 9,84 & $8,11-11,94$ \\
\hline \multirow{2}{*}{$\begin{array}{l}\text { Tipo de horario de } \\
\text { trabajo }\end{array}$} & Normal & 5053 & 53,5 & 8,77 & --- & --- & --- \\
\hline & Atípico & 4390 & 46,5 & 4,19 & $<0,001$ & 0,45 & $0,37-0,54$ \\
\hline \multirow[t]{2}{*}{ Tipo de contrato } & Estable & 3357 & 35,55 & 10,63 & --- & --- & --- \\
\hline & No estable & 6086 & 64,45 & 4,44 & $<0,001$ & 0,39 & $0,32-0,46$ \\
\hline \multirow[t]{3}{*}{ Tipo de actividad } & Escasa & 783 & 8,29 & 1,15 & $<0,001$ & 0,15 & $0,06-0,29$ \\
\hline & Leve -moderada & 4390 & 46,48 & 4,26 & $<0,001$ & 0,59 & $0,46-0,76$ \\
\hline & Moderada-intensa & 4270 & 45,22 & 10,16 & $<0,001$ & 2,91 & $2,44-3,49$ \\
\hline \multirow[t]{4}{*}{ Sistema afectado } & Musculoesquelético & 2217 & 23,47 & 8,12 & $<0,05$ & 3,34 & $2,83-3,78$ \\
\hline & Respiratorio & 3150 & 33,35 & 0,09 & --- & --- & --- \\
\hline & Gastrointestinal & 987 & 10,45 & --- & -- & --- & --- \\
\hline & Otros & 3089 & 32,71 & 4,07 & $<0,05$ & 1,09 & $0,76-1,84$ \\
\hline
\end{tabular}

ALP: Absentismo laboral prolongado, OR: Odds ratio, I.C.: Intervalo de confianza, p: valor p (Chi-Cuadrado)

En relación a la edad, se halló que el grupo que presentó absentismo laboral prolongado tuvo una media de 40,8 años (DS: 12,9), frente a 44,3 años (DS: 12,0) en el grupo de absentismo laboral corto, $(p<0,01)$. El tiempo de servicio en la empresa fue de 10,4 años (DS: 14,6) para el grupo que presentó absentismo laboral prolongado y 15,2 años (DS: 13,7) para absentismo laboral corto, $(p<0,01)$ (Tabla 2, figura 2). 
Tabla 2. Medias y desviación estándar de edad y tiempo de servicio según tipo de absentismo laboral

$\begin{array}{cccc}\begin{array}{c}\text { Variables } \\ \text { continuas }\end{array} & \begin{array}{c}\text { Absentismo corto } \\ (\mathbf{n = 8 8 1 6 )} \\ \text { Media } \pm \text { DS }\end{array} & \begin{array}{c}\text { Absentismo prolongado } \\ (\mathbf{n = 6 2 7}) \\ \text { Media } \pm \text { DS }\end{array} & \mathbf{p} \\ \text { Edad } & 44,3 \pm 12,0 & 40,8 \pm 12,9 & <\mathbf{0 . 0 0 1} \\ \text { Tiempo de servicio } & 15,2 \pm 13,72 & 10,4 \pm 14,6 & <\mathbf{0 . 0 0 1}\end{array}$

DS: Desviación estándar, $\mathrm{p}$ : valor $\mathrm{p}$ (t-Student)

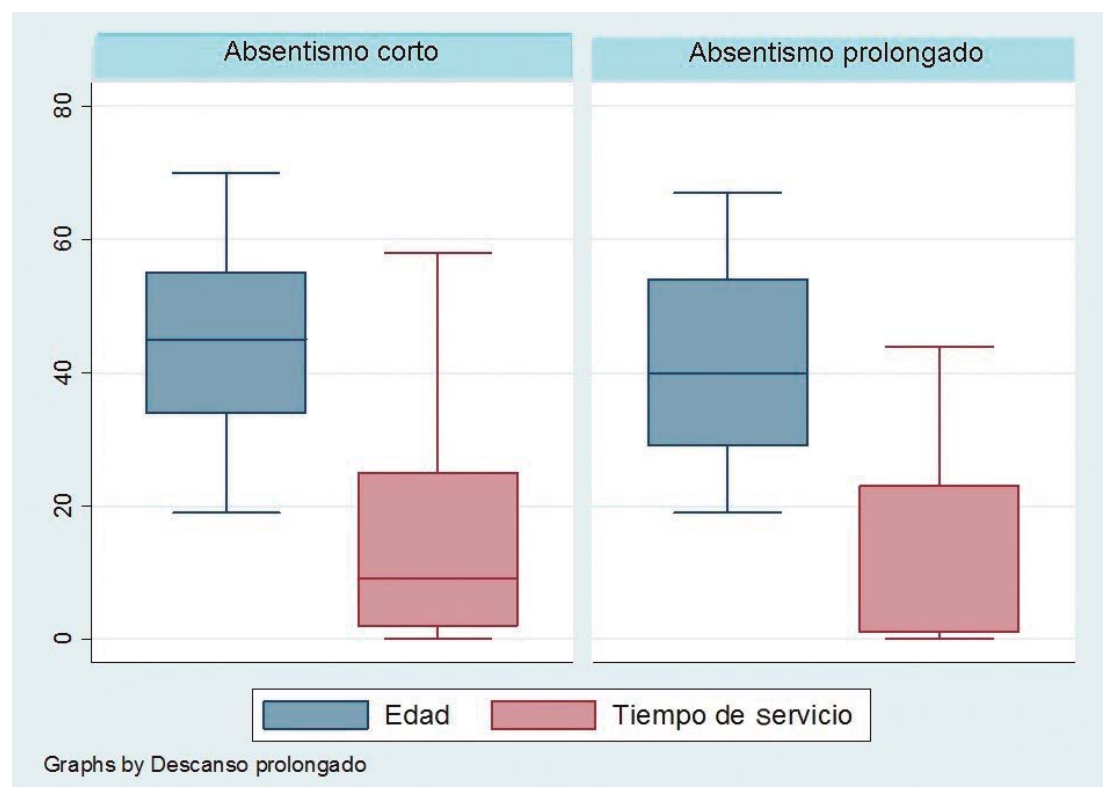

Figura 2. Distribución intercuartílica del tipo de absentismo laboral de causa médica según edad y tiempo de servicio

Al estratificar el tiempo de servicio, se halló que presentar menos de 15 años de servicio es un factor de riesgo para absentismo prolongado comparado a tiempos de servicio mayores (OR: 2,04; IC: 1,7-2,46); mientras que tener entre 15-30 años de servicio no constituye un factor de riesgo para absentismo laboral prolongado (OR: 0,35; IC: 0,27-0,45).

En relación a la contingencia que produjoel absentismo laboral, del total, el $86,3 \%$ (8124) correspondía a enfermedades comunes, 6,82\% (644) a accidentes comunes y 7,15\% (675) a accidentes de trabajo. Quienes presentaron enfermedad común, solo 5,2 \% fueron absentismo prolongado, mientras que los que presentaron accidentes de trabajo, 32,4\% fueron absentismo prolongado, resultado que lo hace evidente como factor de riesgo frente a otras causas (OR: 9,84, I.C. $8,11-11,94)$. Del grupo de accidentes comunes, ninguno tuvo absentismo prolongado.
Respecto al tipo de horario laboral, $46,5 \%$ tuvo un horario de trabajo atípico y de este grupo, solo el 4,19\% presentó absentismo prolongado, frente a $8,77 \%$ en el grupo de los que tenían horarios normales (OR: 0,45, IC: 0,37-0,54).

En relación al tipo de contrato, $35,5 \%$ (3357) tuvo contrato estable; de estos, solo $10,6 \%$ presentó absentismo prolongado, frente a $4,4 \%$ en el grupo de contrato inestable (OR: 0,39; IC: 0,32-0,46).

En relación al tipo de actividad realizada, la actividad física moderada-intensa representa un factor de riesgo frente a otro tipo de actividad (OR: 2,91; IC: 2,44-3,49). La actividad física escasa no resultó ser un factor de riesgo (OR: 0,15; I.C. 0,06-0,29).

Respecto al sistema afectado según diagnóstico, únicamente el compromiso del sistema 
musculoesquelético resultó ser factor de riesgo asociado al absentismo prolongado (OR: 3,34, I.C. $2,83-3,78$ ) (Tabla 1).

Al realizar el análisis multivariado se pudo ver que las variables tiempo de servicio (OR: 0,95 ; I.C. $0,95-0,96$ ), horarios de trabajo atípicos (OR: 3,14; I.C. 1,57-6,24), actividad física moderada-intensa (OR: 8,96; IC: 4,53 17,69), accidentes de trabajo (OR: 13,91; IC: 11,19$17,28)$ y contrato de trabajo no estable (OR: 0,64 ; IC: $0,53-0,76)$ son los factores de riesgo que mejor predicen a la variable resultado (Tabla 3 ).

Tabla 3. Análisis multivariado de los factores asociados al absentismo laboral prolongado de causa médica

\begin{tabular}{|lccc|}
\hline \multicolumn{1}{|c}{ Factores asociados } & O.R. & P & I.C. (95 \%) \\
\hline Tiempo de servicio (mayor de 15 años) & 0,95 & $<0,001$ & $0,95-0,96$ \\
\hline Horario de trabajo (atípico) & 3,14 & 0,001 & $1,57-6,24$ \\
\hline Tipo de actividad (moderada-intensa) & 8,96 & $<0,001$ & $4,53-17,69$ \\
Contingencia (accidente de trabajo) & 13,91 & $<0,001$ & $11,19-17,28$ \\
\hline Tipo de contrato (no estable) & 0,64 & $<0,001$ & $0,53-0,76$
\end{tabular}

OR: Odds ratio ajustado, p: valor p, IC: intervalo de confianza

Respecto al porcentaje global correctamente clasificado se obtuvo un valor del $92,99 \%$ que asegura que el modelo final obtenido es un buen modelo predictor para el tipo de absentismo.

\section{DISCUSIÓN}

Los resultados muestran que los accidentes de trabajo, la realización de actividad física moderada-intensa (manipulación de cargas) y tener horarios de trabajo atípicos constituyen los factores más importantes en el absentismo prolongado de causa médica, y son también factores relevantes el tiempo de servicio y la inestabilidad laboral.

A pesar de que el absentismo laboral debido a afectación del sistema musculoesquelético mostró ser un factor de riesgo evidente respecto a la prolongación de los días de absentismo según el análisis bivariado, en el análisis multivariado fue descartado por presentar colinealidad con la variable accidentes de trabajo, por lo que se eliminó del modelo final por no ser significativo (Tabla 3 ). Esto se debería a que un gran porcentaje de lesiones musculoesqueléticas son producidas por los accidentes de trabajo. El estudio realizado por Ceccato et al. en Brasil muestra resultados similares, con una prevalencia de enfermedades musculoesqueléticas de $29,4 \%$ en mujeres y $36,9 \%$ en hombres. Resultado similar se observó en el grupo con edad menor de 45 años, el cual, en el análisis bivariado mostró que es un factor de riesgo para absentismo prolongado, al respecto se ha reportado resultados similares por Navarro et al. (19) donde las edades entre 35 y 54 años presentan menos ausentismo respecto a edades inferiores. Hoffmeister et al. también presenta datos similares ${ }^{(20)}$.

Respecto al tipo de contrato no estable, este no resultó ser un factor de riesgo en ambos análisis, lo que podría deberse a que una menor estabilidad laboral y menos derechos laborales, coaccionan al trabajador a no ausentarse demasiados días, reduce así el tiempo de recuperación de su salud y aumenta las recidivas de la enfermedad. Esta relación ha sido descrita también por Navarro et al.(19) y Llanos et al. (21), por lo que es un factor a tener en cuenta al momento de hacer la evaluación médica en los trabajadores que se reincorporan a su trabajo luego de un proceso patológico.

Del mismo modo, el mayor tiempo de servicio tampoco resultó ser un factor de riesgo respecto al absentismo prolongado. Este es un hallazgo similar al estudio de Navarro et al. (19), en el cual los trabajadores de entre 1 a 5 años presentan menos ausentismo respecto a trabajadores con más tiempo de servicio. Es importante observar que, en relación al horario de trabajo atípico, en el análisis bivariado no se mostró como un factor de riesgo, pero en el modelo de regresión final, este arrojó un OR ajustado de 3,14,por lo que resulta claramente ser un factor de riesgo para absentismo prolongado. 
Datos similares como los del estudio GAZEL y otros ${ }^{(22-24)}$, permiten afirmar que los desórdenes musculoesqueléticos y las lesiones producidas en los accidentes de trabajo, son causa importante de absentismo prolongado. Sin embargo, es notorio que existe un subregistro de las lesiones musculoesqueléticas derivadas de la actividad física laboral debido a los altos porcentajes de estas afecciones en relación a las enfermedades comunes.

El análisis de los factores asociados al absentismo prolongado, de gran importancia para la productividad laboral, constituye un problema de salud pública debido a la gran carga de enfermedad que representa la población laboral en nuestro país y a un inadecuado sistema de vigilancia de la salud de los trabajadores, relacionándose incluso a un mayor riesgo de mortalidad (22), por lo que debería ser abordado con mayor interés.

A nivel preventivo se debe profundizar en las estrategias para reducir a lo mínimamente posible la exposición de los trabajadores agroindustriales a horarios atípicos de trabajo, actividad física intensa, y accidentes de trabajo, los cuales han demostrado tener impacto en el tiempo de recuperación de la salud, y generar un ambiente y unas condiciones óptimas para que las personas se desempeñen laboralmente de manera adecuada, según recomienda la Organización Mundial de la Salud ${ }^{(25)}$.

Finalmente, respecto a las limitaciones del estudio, es necesario manifestar que, si bien se contó con un tamaño muestral grande, los resultados solamente son aplicables y tienen repercusión en actividades laborales del ámbito agroindustrial. Así mismo, debido a que la obtención de datos fue retrospectiva, estos pudieron ser inadecuadamente registrados. Los sesgos producto de los estudios de fuente secundaria tienen una importante influencia en la calidad de los datos y esto pudo repercutir de alguna forma en los resultados encontrados, por lo que se debería plantear posteriormente un diseño analítico longitudinal prospectivo que permita asegurar un mejor análisis de causalidad.

AGRADECIMIENTO: Al área de Salud Ocupacional de la empresa agroindustrial de donde se obtuvieron los datos, sin los cuales no se habría realizado el presente estudio.

\section{REFERENCIAS BIBLIOGRÁFICAS}

1. Organización Internacional del Trabajo [Internet]. Ginebra: Seguridad y salud en el trabajo. 2016. Disponible en: http: // www.ilo.org/global/topics/safety-and-health-at-work/lang-es/index.htm

2. Roelen CAM, Koopmans PC, de Graaf JH, van Zandbergen JW, Groothoff JW. Job demands, health perception and sickness absence. Occup Med (Lond). 2007;57(7):499-504.
3. García JAR. Análisis de los accidentes de trabajo de una empresa de saneamiento en Perú, en el periodo 2003-2013. [Tesis de Grado]. Lima: Universidad Nacional Mayor de San Marcos; 2014.

4. Escalante GLJ. Análisis de costos por ausentismo laboral atribuibles a licencias médicas por enfermedad Hospital Nacional Arzobispo Loayza. 2015 [Tesis para optar el grado de maestra en gerencia de servicios]. Lima: Universidad de San Martin de Porres; 2015.

5. Baldeón P, Carlos J, Navarro Chumbes G, Zavala Giorfino P, Ruiz Gutiérrez F, Gomero Cuadra R, et al. Relación entre factores de riesgo de salud y costos médicos directos en una población trabajadora peruana durante el año 2003. Rev Med Hered. 2006;17(2):90-5.

6. Gutiérrez FR, Baldeón JP, Beltrán RZ, Yesán CL. Prevalencia, impacto en la productividad y costos totales de las principales enfermedades en los trabajadores de un hospital al sur del Perú en el año 2003. Rev Med Hered. 2012;17(1):28-34.

7. Gomero Cuadra R, Llap Yesan C. Absentismo laboral de origen médico en el Hospital Toquepala en el 2001. Rev Med Hered. 2004;15 (2):95-100.

8. Gomero Cuadra R, Llap Yesan C, Calizaya De la Sota L, Silvera Vallejos R. Accidentes fuera del trabajo: análisis en el campamento minero de Toquepala 2002-2003. Rev Med Hered .2005;16(2):107-13.

9. Ceccato ADF, Junior C, De LCS, Cuissi RC, Monteschi M, Oliveira NG, et al. Absenteeism due to occupational diseases among sugarcane workers. Cad Saúde Pública. 2014;30(10):2169-76.

10. Odeen M, Magnussen LH, Maeland S, Larun L, Eriksen HR, Tveito TH. Systematic review of active workplace interventions to reduce sickness absence. Occup Med (Lond). 2013;63(1):7-16.

11. Andrea H, Beurskens A, Metsemakers J, van Amelsvoort LGPM, van den Brandt PA, van Schayck CP. Health problems and psychosocial work environment as predictors of long term sickness absence. Occup Environ Med. 2003;60(4):295300.

12. Laaksonen M, Martikainen P, Rahkonen O, Lahelma E. The effect of occupational and workplace gender composition on sickness absence. J Occup Environ Med. 2012;54(2):224-30.

13. Fumado $M$, Amengual $M$, Bausili $P$, Manso A. Temporary disability and its legal implications. Med Clin (Barc).2014;142 (Suppl 2):37-42.

14. Hansson T, Jensen I. Chapter 6. Sickness absence due to back and neck disorders. Scand J Public Health. 2004;32(suppl 63):109-51.

15. Trespalacios V, María E. Absentismo laboral por causa médica en trabajadores del área operativa de una compañía de extracción de minerales en Colombia, 2011. Med Segur Trab. 2013;59(230):93-101.

16. Godin I, Kittel F. Differential economic stability and psychosocial stress at work: associations with psychosomatic complaints and absenteeism. Soc Sci Med.2004;58(8):154353.

17. Rajaratnam SMW, Licamele L, Birznieks G. Delayed sleep phase disorder risk is associated with absenteeism and impaired functioning. Sleep Health. 2015;1(2):121-7.

18. Burton WN, Chen C-Y, Schultz AB, Li X. Association Between Employee Sleep With Workplace Health and Economic Outcomes. J Occup Environ Med. 2017;59(2):177-83. 
19. Navarro A, Salas-Nicás S, Moncada S, Llorens C, Molinero-Ruiz E. Prevalence, associated factors and reasons for sickness presenteeism: a cross-sectional nationally representative study of salaried workers in Spain, 2016. BMJ Open. 2018;8(7):e021212.

20. Hoffmeister L, Vidal C, Vallebuona C, Ferrer N, Vásquez P, Núñez G. Factores Asociados a Accidentes, Enfermedades y Ausentismo Laboral: Análisis de una Cohorte de Trabajadores Formales en Chile. Cienc Trab. 2014; 16(49):21-7.

21. Mendoza Llanos R. Insatisfacción laboral como predictor del ausentismo en un hospital público. Rev Med Chil. 2015 ;143(8):1028-33.

22. Ferrie JE, Vahtera J, Kivimäki $M$, Westerlund $H$, Melchior $M$, Alexanderson $\mathrm{K}$, et al. Diagnosis-specific sickness absence and all-cause mortality in the GAZEL study. J Epidemiol Community Health. 2009;63(1):50-5.

23. Mesa M, Francisco R, Kaempffer R, María A. 30 años de estudio sobre ausentismo laboral en Chile: una perspectiva por tipos de empresas. Rev Med Chil. 2004; 132(9):1100-8.

24. Black CM. Sickness absence and musculoskeletal disorders. Rheumatology (Oxford). 2012;51(2):204-5.

25. WHO Healthy workplaces [Internet]. Geneva: a WHO global model for action. 2017. Disponible en: http://www.who. int/occupational_health/healthy_workplaces/en/
Fuentes de financiamiento:

Este artículo ha sido financiado por los autores.

Conflictos de interés:

Los autores declaran no tener ningún conflicto de interés.

\section{Correspondencia:}

Wilfor Aguirre Quispe

Dirección: Jr Sinchi Roca 2287, Lince. Lima, Perú.

Teléfono: 941925104

Correo electrónico: wilfor.aguirre.q@upch.pe

$\begin{array}{llll}\text { Recibido: } & 09 & \text { de julio de } & 2018 \\ \text { Evaluado: } 30 \text { de julio de } 2018 \\ \text { Aprobado: } 28 \text { de agosto de } 2018\end{array}$

(c) La revista. Publicado por Universidad de San Martín de Porres, Perú. (c) $\mathbf{B r}$ Licencia de Creative Commons Artículo en acceso abierto bajo términos de Licencia Creative Commons Atribución 4.0 Internacional. (http://creativecommons.org/licenses/by/4.0/)

ORCID iDs

Wilfor Aguirre Quispe

Joan Manuel Moreno Luján

https://orcid.org/0000-0002-6677-0900

https://orcid.org/0000-0003-2621-7198 